\title{
Sarcopenia in COPD: prevalence, clinical correlates and response to pulmonary rehabilitation
}

\author{
Sarah E Jones, ${ }^{1}$ Matthew Maddocks, ${ }^{2}$ Samantha S C Kon, ${ }^{1}$ Jane L Canavan, ${ }^{1}$ \\ Claire M Nolan, ${ }^{1,3}$ Amy L Clark, ${ }^{3}$ Michael I Polkey, ${ }^{1}$ William D-C Man ${ }^{1,3}$
}

\begin{abstract}
- Additional material is published online only. To view please visit the journal online (http://dx.doi.org/10.1136/ thoraxjn-2014-206440)
\end{abstract}

${ }^{1}$ NIHR Respiratory Biomedical Research Unit, Royal Brompton \& Harefield NHS Foundation Trust and Imperial College, London, UK

${ }^{2}$ King's College London, Cicely Saunders Institute, London, UK ${ }^{3}$ Harefield Pulmonary Rehabilitation Unit, Royal Brompton and Harefield NHS Foundation Trust, London, UK

\section{Correspondence to} Dr Matthew Maddocks, Lecturer in Health Service Research, King's College London, Cicely Saunders Institute, London SE5 9PJ, UK; matthew.maddocks@kcl.ac.uk

SEJ and MM contributed equally.

Received 10 October 2014 Revised 24 November 2014 Accepted 2 December 2014 Published Online First 5 January 2015

\section{ABSTRACT \\ Background Age-related loss of muscle, sarcopenia, is recognised as a clinical syndrome with multiple contributing factors. International European Working Group on Sarcopenia in Older People (EWGSOP) criteria require generalised loss of muscle mass and reduced function to diagnose sarcopenia. Both are common in COPD but are usually studied in isolation and in the lower limbs.}

Objectives To determine the prevalence of sarcopenia in COPD, its impact on function and health status, its relationship with quadriceps strength and its response to pulmonary rehabilitation (PR).

Methods EWGSOP criteria were applied to 622 outpatients with stable COPD. Body composition, exercise capacity, functional performance, physical activity and health status were assessed. Using a case-control design, response to PR was determined in 43 patients with sarcopenia and a propensity score-matched non-sarcopenic group.

Results Prevalence of sarcopenia was $14.5 \%(95 \% \mathrm{Cl}$ $11.8 \%$ to $17.4 \%$ ), which increased with age and Global Initiative for Chronic Obstructive Pulmonary Disease (GOLD) stage, but did not differ by gender or the presence of quadriceps weakness ( 14.9 vs $13.8 \%, p=0.40$ ). Patients with sarcopenia had reduced exercise capacity, functional performance, physical activity and health status compared with patients without sarcopenia $(p<0.001)$, but responded similarly following PR; $12 / 43$ patients were no longer classified as sarcopenic following PR.

Conclusions Sarcopenia affects 15\% of patients with stable COPD and impairs function and health status. Sarcopenia does not impact on response to PR, which can lead to a reversal of the syndrome in select patients.

\section{INTRODUCTION}

Skeletal muscle dysfunction is a recognised manifestation of COPD. ${ }^{1}$ Key features include quadriceps weakness, ${ }^{2}$ atrophy ${ }^{3}$ and a type II fibre shift ${ }^{4}$ all of which are associated with a poor prognosis independent of lung function. ${ }^{5-7}$ Sarcopenia describes age-related loss of skeletal muscle, which leads to increased risk of physical disability, poor health status and death. ${ }^{8}{ }^{9}$ Sarcopenia is increasingly recognised as a clinical syndrome with multiple contributing factors, including physical inactivity, malnutrition and chronic disease. Since COPD, in some respects, is considered a disease of accelerated ageing, one would hypothesise that sarcopenia would be relevant to patients with COPD.

In COPD, most studies examining dysfunction of skeletal muscle have focused on one aspect of

\section{Key Messages}

What is the key question?

- What is the prevalence and impact of sarcopenia in COPD and does sarcopenia affect response to pulmonary rehabilitation?

\section{What is the bottom line?}

- Sarcopenia affects $15 \%$ of patients with stable COPD and impairs exercise capacity, functional performance and health status, though it does not limit response to pulmonary rehabilitation.

\section{Why read on?}

- We characterise the sarcopenia phenotype in COPD using internationally agreed criteria and demonstrate the impact of pulmonary rehabilitation, which can reverse the syndrome in select patients.

sarcopenia, predominantly in the lower limbs. ${ }^{10}$ This contrasts with international consensus statements for sarcopenia, which emphasise the loss of both muscle mass and function in diagnostic criteria, and underscore the importance of generalised muscle dysfunction. ${ }^{8}$ In particular, assessment of a single aspect of sarcopenia is considered insufficient, as the relationship between muscle mass and strength is non-linear, ${ }^{3}$ because muscle atrophy does not always lead to impairment of function ${ }^{11}$ and cut points for weakness that relate to functional status are lacking. ${ }^{12}$

The European Working Group on Sarcopenia in Older People (EWGSOP) developed practical clinical diagnostic criteria for sarcopenia ${ }^{8}$ which are endorsed by international organisations and have been used to examine the prevalence and impact of the syndrome across settings ${ }^{12-14}$ and disease states. ${ }^{9}$ Though the term has been used loosely in COPD,${ }^{15}$ data on consensus-defined sarcopenia are lacking, but are necessary to understand the size and nature of this problem in a disease characterised by differential muscle atrophy and weakness. Sarcopenia is relevant to many common disease management strategies, including exercise training $^{10}$ and nutritional therapy. ${ }^{16}$ Given the advent of medicines directed at sarcopenia in other disease conditions, such data will also be useful for drug development. ${ }^{17}$

In this study, we used the EWGSOP criteria to determine the prevalence and risk factors for 
sarcopenia in COPD and to determine the impact of sarcopenia on functional exercise capacity and health status. We also sought to examine the relationship between sarcopenia and quadriceps strength and explore if sarcopenia can be reversed by exercise training as part of pulmonary rehabilitation (PR).

\section{METHODS}

\section{Participants}

Patients diagnosed with COPD according to Global Initiative for Chronic Obstructive Pulmonary Disease (GOLD) guidelines were recruited from outpatient respiratory clinics at Harefield Hospital (Harefield, Middlesex, UK) between April 2011 and January 2014. Exclusion criteria were unstable cardiac disease, an exacerbation within the preceding 4 weeks, predominant neurological limitation to walking (eg, significant hemiplegia) or contraindication to bioelectrical impedance analysis (BIA) including an implanted pacemaker or defibrillator. All participants gave written informed consent.

\section{Assessments}

Sarcopenia was defined according to the EWGSOP criteria. ${ }^{8}$ Skeletal muscle mass (SMM, kg) was estimated using wholebody BIA and Skeletal Muscle Mass Index (SMI) calculated as $\mathrm{SMM} /$ height $^{2}$. Handgrip strength was measured using a handheld dynamometer and physical performance by the 4-metre gait speed (4MGS), as previously described. ${ }^{18} 19$ The following cut-off values were used to identify sarcopenia: SMI of $\leq 8.50 \mathrm{~kg} / \mathrm{m}^{2}$ for men and $\leq 5.75 \mathrm{~kg} / \mathrm{m}^{2}$ for women, ${ }^{20}$ and either handgrip strength of $<30 \mathrm{~kg}$ for men and $<20 \mathrm{~kg}$ for women, or a gait speed of $<0.8 \mathrm{~m} / \mathrm{s}^{8}{ }^{21}$

Further measurements included the incremental shuttle walk test (ISWT), five-repetition sit-to-stand test (5STS), short physical performance battery (SPPB), quadriceps maximum voluntary contraction (QMVC), St George's Respiratory Disease Questionnaire and COPD Assessment Test. Physical activity was assessed by the modified Minnesota Leisure-time Physical Activity Questionnaire and, in a subgroup, by a multisensory accelerometer (SenseWear, Bodymedia, Pittsburgh, USA) which integrates biaxial accelerometry (longitudinal and transverse planes) with multiple physiological measures including galvanic skin resistance and body temperature to calculate estimates of energy expenditure. ${ }^{22}$ The iBODE composite prognostic index (Body mass index, airflow Obstruction, Dyspnoea, and Exercise capacity) was calculated. Comorbidity burden was assessed by the Charlson index. Full details and references are found in the online supplement.

\section{Pulmonary rehabilitation}

The PR programme was an 8 -week outpatient multidisciplinary exercise and education programme comprising two supervised and at least one additional home session per week. Further details of exercise prescription and educational content are found in the online supplement.

\section{Statistical analysis}

Data are presented as mean (SD) or median (25th, 75 th percentiles) where data were not normally distributed. The prevalence of sarcopenia with 95\% CIs was determined and compared according to gender, age, GOLD staging, iBODE and quadriceps weakness ${ }^{2}$ using the $\chi^{2}$ for trend test with posthoc comparisons between a reference group with the lowest prevalence. Baseline characteristics were compared between groups according to the presence of sarcopenia, or low SMI, handgrip strength, or
4MGS alone using one-way analysis of variance or KruskalWallis tests with posthoc comparisons.

Response to PR was calculated for patients with sarcopenia who had attended $\geq 50 \%$ of the supervised sessions $(\geq 8 / 16)$ and had completed a post-PR assessment. A matched group of patients without sarcopenia fulfilling these criteria were identified by using propensity score matching, ${ }^{23}$ considering baseline age, gender, $\mathrm{FEV}_{1} \%$ predicted, Medical Research Council dyspnoea score, and ISWT distance as moderators of PR outcome. Change from pre-PR to post-PR was compared between groups using independent $t$ test or Mann-Whitney $U$ test. Analyses were performed using SPSS (V.21, IBM, New York, USA). To control for Type I errors in view of multiple testing, a $\mathrm{p}$ value $<0.01$ was considered significant.

\section{RESULTS}

\section{Prevalence of sarcopenia in COPD}

Six hundred and twenty-two outpatients with stable COPD were studied. The overall prevalence of sarcopenia was $14.5 \%$ (11.8\% to $17.4 \%)$. A flowchart, according to the EWGSOP

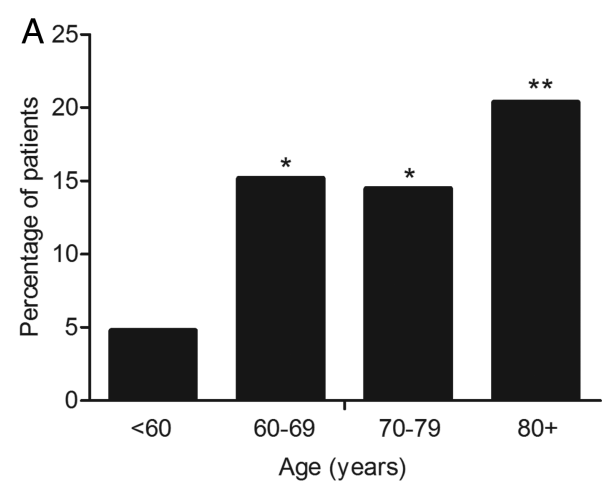

B

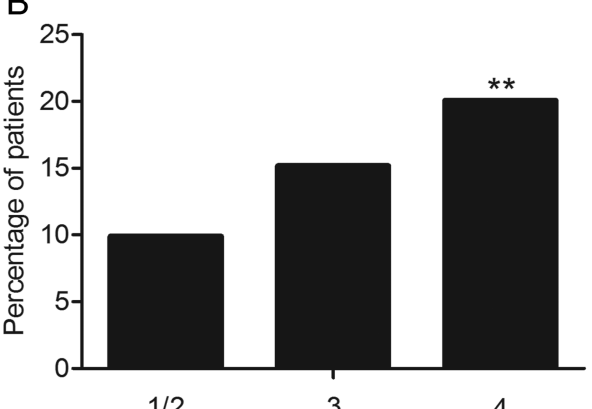

$1 / 2$

GOLD stage

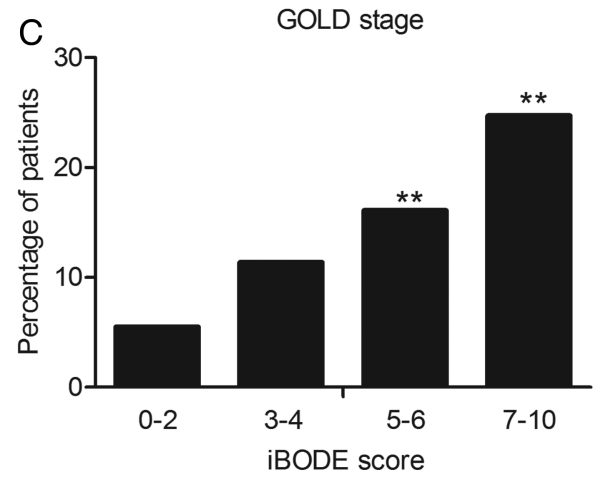

Figure 1 Prevalence of sarcopenia according to age (A), disease severity (B), and Body mass index, obstruction, dyspnoea, exercise capacity index (BODE) (C). Between-group differences compared with base group (far left) denoted by ${ }^{*}<0.05$ or ${ }^{*}<0.01$. 
algorithm, is outlined in online supplementary figure S1. Prevalence did not differ by gender (men 16.1\% $(12.4 \%$ to $20.3 \%)$ vs women $12.3 \%(8.6 \%$ to $16.9 \%)$; $\mathrm{p}=0.20)$, but increased with age $(\mathrm{p}=0.020)$ (figure 1A) and GOLD stage $(p=0.005)$, with a twofold increase in stage IV, 20.1\% $(14.2 \%$ to $27.2 \%)$ as compared with stage I/II disease, $9.9 \%(6.4 \%$ to $14.5 \%$ ) (figure 1B). Prevalence also increased with iBODE score $(\mathrm{p}<0.001)$ and was lowest in the quartile with the best prognosis (iBODE 0-2: $5.5 \%(2.4 \%$ to $10.5 \%)$ ) and highest in the quartile with the worst prognosis (iBODE 7-10: 24.7\% (18.1\% to $32.3 \%)$ ) (figure $1 \mathrm{C}$ ).

\section{Factors associated with sarcopenia in COPD}

Baseline clinical characteristics, according to the presence of low SMI, low physical function or sarcopenia, are shown in table 1. About one-third of the patients met none of the criteria and over half of the patients met one of the criteria, most often low physical function (figure 2A). Low muscle mass alone impacted adversely on 5STS but not on other outcomes of physical functions, such as 4MGS, ISWT or SPPB, or on self-reported physical activity (table 1). Patients with sarcopenia were significantly older, had more airflow obstruction and reduced quadriceps strength, exercise capacity, functional performance, subjective and objective physical activity and health status compared with patients without sarcopenia. There were no significant differences in smoking status, number of comorbidities, self-reported hospital admissions or exacerbations between groups (table 1).

Of the 622 patients, 554 patients had QMVC measured. When patients were classified according to quadriceps weakness, there was no significant difference in the prevalence of sarcopenia between groups (weak: $14.9 \%(11.2 \%$ to $18.6 \%)$ vs normal: $13.8 \%(10.2 \%$ to $18.0 \%), \mathrm{p}=0.404)$. Furthermore, of the 90 patients who were sarcopenic, $33(36.7 \%)$ did not present with quadriceps weakness (figure 2B). Clinical characteristics, according to the presence of quadriceps weakness, sarcopenia or both, are found in the online supplementary table S1.

\section{Response to PR}

Patients with and patients without sarcopenia attended a mean (SD) of 13 (2) and 13 (3) PR sessions, respectively. Following PR, there were no statistical differences for change in outcomes of body composition, functional performance or health status between patients with and patients without sarcopenia (table 2). In the sarcopenic group, SMI, handgrip strength and 4MGS improved by a mean of $0.11 \mathrm{~kg} / \mathrm{m}^{2}, 2.08 \mathrm{~kg}$ and $0.12 \mathrm{~m} / \mathrm{s}$, respectively, such that $12 / 43$ patients $(28 \%)$ no longer met EWGSOP criteria for sarcopenia. Adherence was similar in patients whose state was reversed and unchanged (14 (2) vs 13 (2) sessions). Three

Table 1 Baseline clinical characteristics expressed as $\mathrm{n} / \%$, mean (SD) and median (25th and 75 th centiles)

\begin{tabular}{|c|c|c|c|c|c|}
\hline & No sarcopenia $(n=220)$ & Low SMI only $(n=27)$ & Low function only $(n=285$ ) & Sarcopenia $(n=90)$ & p Value \\
\hline Age (years) & $66(10)$ & $69(7)$ & $73(9)^{*}$ & $73(8)^{*}$ & $<0.001$ \\
\hline Sex (M:F) & $147: 73$ & $14: 13$ & $136: 149 *$ & $57: 33$ & $<0.001$ \\
\hline MRC & $3(1)$ & $3(1)$ & $4(1)^{*}$ & $4(1)^{*}$ & $<0.001$ \\
\hline FEV1 (\% predicted) & $46.3(18.6)$ & $37.9(16.6)$ & $45.6(17.7)$ & $40.5(19.6)$ & 0.013 \\
\hline LTOT (n/\%) & $12 / 5$ & $1 / 4$ & $19 / 7$ & $4 / 9$ & 0.811 \\
\hline Use of walking aid (n/\%) & $5 / 2$ & $0 / 0$ & $54 / 19^{*}$ & $15 / 17^{*}$ & $<0.001$ \\
\hline BMI $(\mathrm{kg} / \mathrm{m})$ & $28.8(5.7)$ & $21.1(3.0)^{*}$ & $29.2(6.2)$ & $21.4(4.0)^{*}$ & $<0.001$ \\
\hline SMM (kg) & $26.6(6.9)$ & $18.8(5.0)^{*}$ & $22.9(6.5)$ & $18.9(5.1)^{*}$ & $<0.001$ \\
\hline SMI $\left(\mathrm{kg} / \mathrm{m}^{2}\right)$ & $9.2(1.8)$ & $6.8(1.4)^{*}$ & $8.6(1.8)^{*}$ & $6.9(1.3)^{*}$ & $<0.001$ \\
\hline Handgrip (kg) & $33.9(8.6)$ & $29.6(7.5)$ & $22.6(8.4)^{*}$ & $21.5(7.3)^{*}$ & $<0.001$ \\
\hline Peak QMVC (kg) & $32.2(10.5)$ & $23.0(7.6)^{*}$ & $23.3(8.2)^{*}$ & $19.8(7.6)^{*}$ & $<0.001$ \\
\hline QMVC $\%$ predicted & $70.1(17.0)$ & $63.1(14.4)$ & $56.3(14.3)^{*}$ & $54.9(16.3)^{*}$ & $<0.001$ \\
\hline 4MGS $(\mathrm{m} / \mathrm{s})$ & $1.07(0.16)$ & $1.00(0.16)$ & $0.77(0.21)^{*}$ & $0.77(0.22)^{*}$ & $<0.001$ \\
\hline 5STS (s) & $12.4(10.4,14.8)$ & $14.1(11.4,13.9)$ & $16.1(12.8,26.7)^{*}$ & $19.6(12.9,60.0)^{*}$ & $<0.001$ \\
\hline SPPB & $11(1)$ & $11(1)$ & $8(3)^{*}$ & $8(3)^{*}$ & $<0.001$ \\
\hline ISWT (m) & $309(153)$ & $257(99)$ & $152(106)^{*}$ & $157(118)^{*}$ & $<0.001$ \\
\hline CAT & $19(7)$ & $22(7)$ & $23(8)^{*}$ & $24(9)$ * & $<0.001$ \\
\hline SGRQ total & $47.3(16.1)$ & $51.0(14.0)$ & $55.9(17.1)^{*}$ & $57.1(17.5)$ * & $<0.001$ \\
\hline Smoking status (current:former:never) & $43: 170: 7$ & $6: 19: 2$ & $49: 216: 20$ & $16: 67: 7$ & 0.561 \\
\hline Charlson Index & $1(1,2)$ & $1(1,3)$ & $1(1,3)$ & $1(1,2)$ & 0.151 \\
\hline Hospital inpatient days previous 12 months & $0(0,2)$ & $0(0,2)$ & $0(0,5)$ & $0(0,4)$ & 0.791 \\
\hline Number of exacerbations previous 12 months & $2(1,3)$ & $2(1,5)$ & $2(1,4)$ & $2(1,4)$ & 0.128 \\
\hline \multicolumn{6}{|l|}{ Self-report physical activity } \\
\hline Energy expenditure (kcal/week) & $780.3(388.1,1567.5)$ & $695.6(288.8,1506.3)$ & $380.0(89.1,868.1)^{*}$ & $376.3(56.9,734.4)^{*}$ & $<0.001$ \\
\hline Time in moderate activity (min/week) & $224.0(108 \cdot 8,450.6)$ & $193.1(82.5,432.5)$ & $110.3(27.3,241.9)^{*}$ & $107.5(22.5,207.5)^{*}$ & $<0.001$ \\
\hline \multicolumn{6}{|l|}{ Objective physical activity $(n=129)$} \\
\hline Daily step count & $5127(2475,6815)$ & $3916(1679,5411)$ & $2697(1129,4995)$ & $1482(1205,3301)$ & $<0.001$ \\
\hline Physical activity level (METS/day) & $1.45(0.22)$ & $1.60(0.21)$ & $1.38(0.85)$ & $1.36(0.12)$ & 0.034 \\
\hline Time in moderate intensity activity $\geq 3$ METS (min/day) & $47(20,106)$ & $108(63,223)$ & $30(8,69)$ & $35(5,82)$ & 0.018 \\
\hline Active energy expenditure (kcal/day) & $246(106,473)$ & $466(219,847)$ & $134(42,329)$ & $105(25,276)$ & 0.006 \\
\hline
\end{tabular}

*Significant difference to 'no sarcopenia' reference group.

4MGS, 4-metre gait speed; 5STS, five-repetition sit-to-stand test; BMI, body mass index; CAT, COPD Assessment Test; iBODE, body mass index, obstruction, dyspnoea, exercise capacity Index; ISWT, incremental shuttle walk test; kcal, kilocalorie; LTOT, long-term oxygen therapy; METS, metabolic equivalents; MRC, Medical Research Council; SGRQ, St George's

Respiratory Disease Questionnaire; SMI, Skeletal Muscle mass Index; SMM, skeletal muscle mass; SPPB, short physical performance battery; QMVC, quadriceps maximum voluntary contraction. 

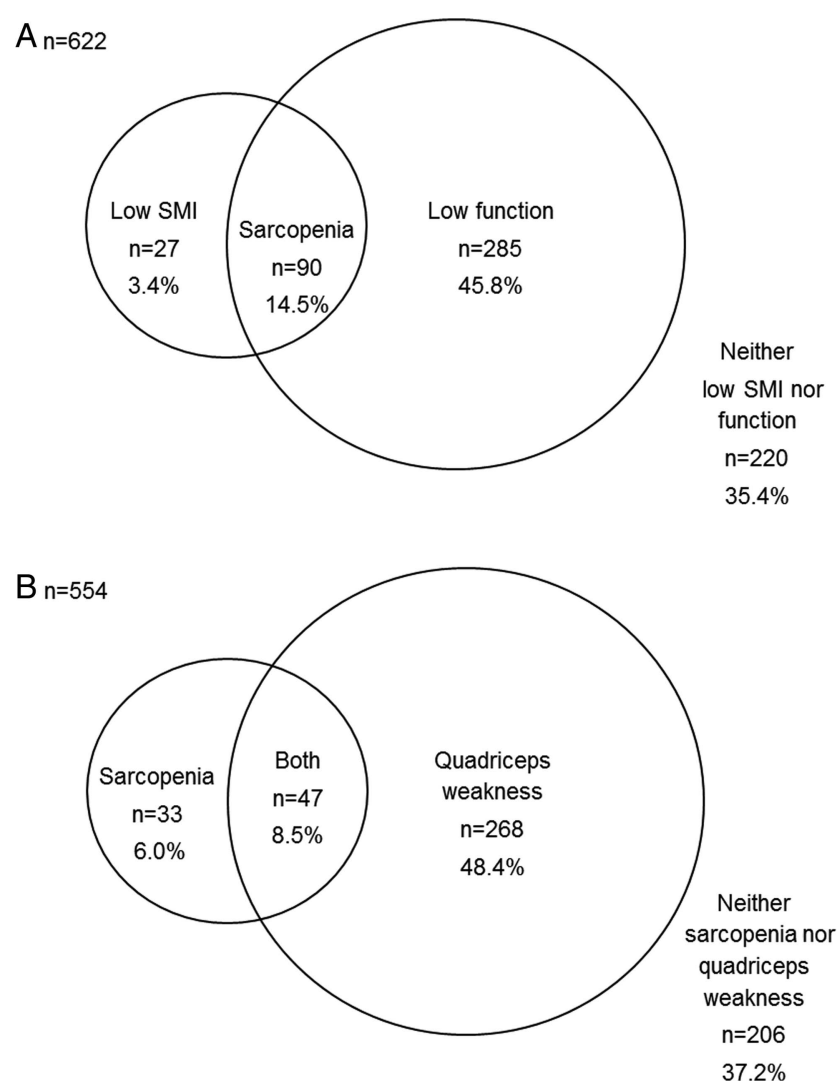

Figure 2 Relationships between sarcopenia and (A) low muscle mass or function, and (B) quadriceps weakness. patients had a change in both mass and functional status, six patients had a change in SMI only and three patients changed their functional status (one improved gait speed and two improved handgrip strength). These patients were generally closer to cut-offs for SMI and function at baseline (figure 3).

\section{DISCUSSION}

We have identified a $14.5 \%$ prevalence of sarcopenia in patients with stable COPD as defined by EWGSOP criteria, ${ }^{8}$ which increased with age and disease severity. Sarcopenia was distinct from the loss of quadriceps strength alone and was associated with reduced functional performance, exercise capacity and quality of life. Despite this, sarcopenia did not appear to impact on response to PR. In about one-quarter of patients, PR completion led to a reversal of their sarcopenia status.

\section{Critique of the method}

Various definitions for sarcopenia have been proposed ${ }^{24}$ including some that only consider muscle mass, as suggested within the recent European Respiratory Society (ERS) statement for nutritional assessment and therapy. ${ }^{16}$ The current international consensus is that the sarcopenia syndrome includes the presence of both low muscle mass and function, hence, our decision to adopt the EWGSOP criteria. ${ }^{8}$ Our study supports the construct validity of these criteria which identified sarcopenic patients as having the greatest levels of impairment across a range of domains. Our large cohort of well-phenotyped patients with stable COPD provides an accurate estimation of overall prevalence of sarcopenia, and allows for detailed study around the effects of sarcopenia on clinical outcome. The use of EWGSOP criteria also permits

Table 2 Comparison of response to pulmonary rehabilitation between sarcopenic and propensity-matched patients with non-sarcopenic COPD expressed as mean $(95 \% \mathrm{Cl})$

\begin{tabular}{|c|c|c|c|c|c|c|}
\hline & \multicolumn{2}{|l|}{ Baseline } & \multirow[b]{2}{*}{ p Value } & \multicolumn{2}{|l|}{ Change with PR } & \multirow[b]{2}{*}{$p$ Value } \\
\hline & Non-sarcopenic & Sarcopenic & & Non-sarcopenic & Sarcopenic & \\
\hline Mean age (years $(S D))^{*}$ & $72(11)$ & $73(8)$ & 0.733 & - & - & - \\
\hline Gender* (M:F) & $25: 18$ & $28: 15$ & 0.508 & - & - & - \\
\hline $\mathrm{FEV}^{1} \%$ predicted ${ }^{*}$ & 45.1 (39.2 to 51.0$)$ & 43.5 (37.4 to 50.2 ) & 0.728 & - & - & - \\
\hline $\mathrm{MRC}^{*}$ & 3 (3.3 to 3.9$)$ & 3 (3.0 to 3.6$)$ & 0.275 & $-0.8(-1.1$ to -0.5$)$ & $-0.7(-1.0$ to -0.3$)$ & 0.495 \\
\hline Weight (kg) & 77.3 (77.0 to 81.4$)$ & 57.9 (53.9 to 62.2) & $<0.001$ & $0.0(-0.6$ to 0.6$)$ & $-0.1(-0.5$ to 0.3$)$ & 0.616 \\
\hline BMI $(\mathrm{kg} / \mathrm{m})$ & 28.7 (27.3 to 30.0$)$ & 21.3 (20.1 to 22.5$)$ & $<0.001$ & $0.0(-0.3$ to 0.2$)$ & $0.0(-0.3$ to 0.1$)$ & 0.723 \\
\hline SMM (kg) & 23.9 (22.1 to 25.6$)$ & 19.1 (17.6 to 20.7 ) & $<0.001$ & $0.1(-0.3$ to 0.5$)$ & $0.3(0.0$ to 0.7$)$ & 0.363 \\
\hline SMI $\left(\mathrm{kg} / \mathrm{m}^{2}\right)$ & 8.8 (8.3 to 9.3$)$ & $7.0(6.6$ to 7.3$)$ & $<0.001$ & $0.0(-0.1$ to 0.2$)$ & $0.1(0.0$ to 0.2$)$ & 0.431 \\
\hline Handgrip (kg) & 25.7 (22.8 to 28.4$)$ & $21.2(19.2$ to 23.3$)$ & 0.021 & $1.6(0.5$ to 2.6$)$ & 2.1 (1.3 to 2.9 ) & 0.516 \\
\hline QMVC (kg) & 25.7 (23.0 to 28.3 ) & 19.9 (17.9 to 22.3$)$ & 0.003 & $0.9(-1.1$ to 3.5$)$ & $1.4(0.3$ to 3.7$)$ & 0.646 \\
\hline QMVC \% predicted & $60.0(55.3$ to 64.4$)$ & 55.1 (50.1 to 60.3$)$ & 0.164 & $1.1(-3.7$ to 6.5$)$ & $3.8(0.4$ to 9.8$)$ & 0.574 \\
\hline 4MGS (m/s) & $0.86(0.80$ to 0.91$)$ & 0.83 (0.77 to 0.89$)$ & 0.511 & 0.11 (0.07 to 0.14$)$ & 0.12 (0.08 to 0.16$)$ & 0.552 \\
\hline 5STS (s) & 15.5 (13.6 to 18.4$)$ & 18.1 (13.2 to 28.8$)$ & 0.186 & $-2.9(-4.1$ to -1.6$)$ & $-1.7(-3.9$ to 0.0$)$ & 0.517 \\
\hline SPPB & 9 (8.4 to 9.8$)$ & 9 (8.0 to 9.4$)$ & 0.386 & $1.0(10.51$ to 1.5$)$ & $0.9(0.4$ to 1.4$)$ & 0.746 \\
\hline ISWT $(\mathrm{m})^{*}$ & 168 (139 to 197$)$ & 194 (158 to 232) & 0.284 & $40(30$ to 60$)$ & $50(40$ to 80$)$ & 0.214 \\
\hline CAT & 20.0 (17.5 to 22.2$)$ & 23.8 (21.3 to 26.4$)$ & 0.031 & $-3.0(-4.4$ to -1.5$)$ & $-4.7(-6.9$ to -2.3$)$ & 0.236 \\
\hline \multicolumn{7}{|l|}{ SGRQ } \\
\hline Symptoms & 64.6 (58.3 to 70.9$)$ & $68.5(63.5$ to 74.1$)$ & 0.360 & $-9.7(-17.3$ to -2.4$)$ & $-7.85(-12.0$ to -3.6$)$ & 0.675 \\
\hline Activity & 71.8 (64.6 to 79.1$)$ & 73.7 (68.4 to 78.9$)$ & 0.685 & $-5.7(-10.4$ to -1.5$)$ & $-5.66(-10.7$ to -0.4$)$ & 0.994 \\
\hline Impact & 38.1 (32.0 to 44.5$)$ & 39.9 (34.3 to 45.7$)$ & 0.668 & $-7.9(12.0$ to -3.7$)$ & $-7.27(-10.8$ to -3.7$)$ & 0.821 \\
\hline Total & 52.7 (47.3 to 58.4$)$ & $54.9(50.1$ to 60.1$)$ & 0.570 & $-6.3(-9.5$ to -3.0$)$ & $-6.81(-10.1$ to -3.4$)$ & 0.836 \\
\hline
\end{tabular}

*Variable used for propensity score.

4MGS, 4-metre gait speed; 5STS, five-repetition sit-to-stand test: BMI, Body Mass Index; CAT, COPD Assessment Test; ISWT, incremental shuttle walk test; MRC, Medical Research Council; PR, pulmonary rehabilitation; SGRQ, St George's Respiratory Disease Questionnaire; SMI, Skeletal Muscle Mass Index; SMM, skeletal muscle mass; SPPB, short physical performance battery; QMVC, quadriceps maximum voluntary contraction. 

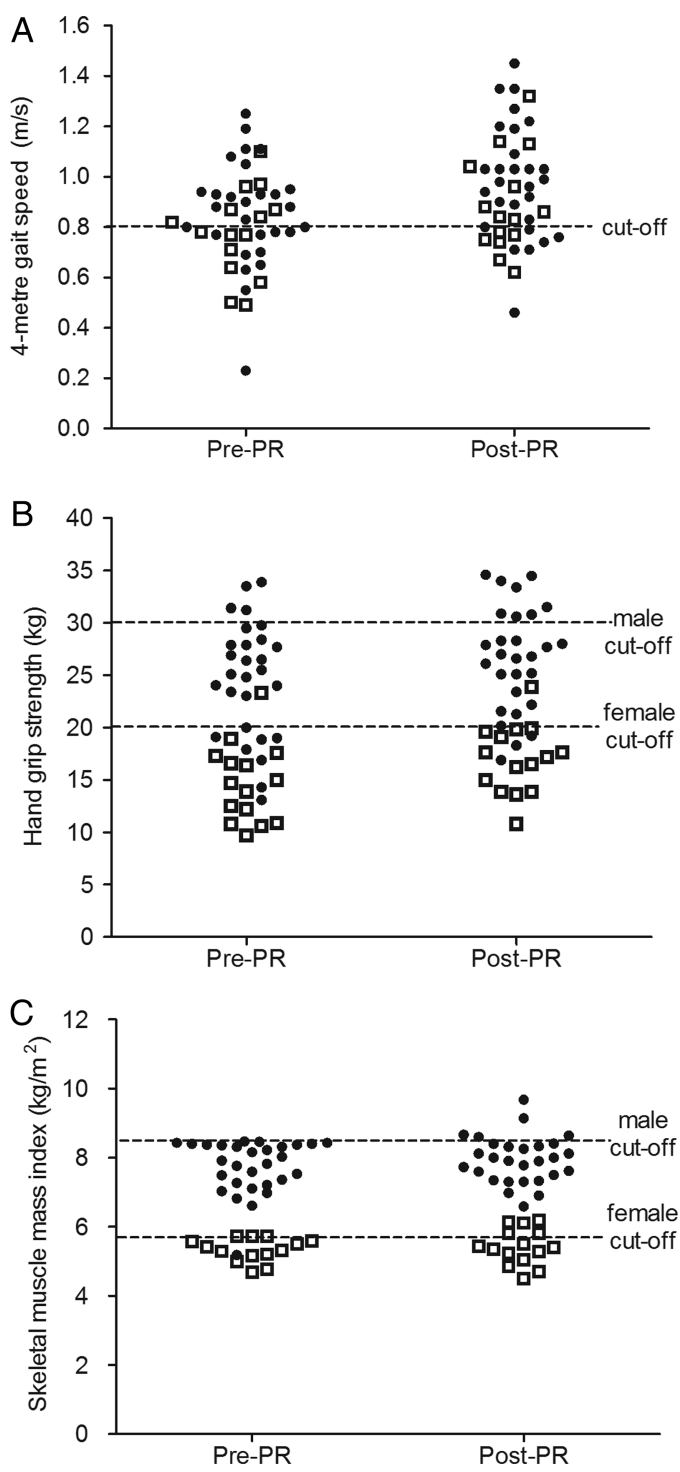

Figure 3 Response to pulmonary rehabilitation (PR) in sarcopenic male (closed circles) and female (open squares) patients according to European Working Group on Sarcopenia in Older People criteria for diagnosing sarcopenia. SMI, Skeletal Muscle Mass Index.

comparison of prevalence and characteristics of a sarcopenic phenotype in COPD with other patient groups.

We acknowledge that this study was undertaken in ambulatory hospital outpatients, and hence, our findings need to be corroborated in other COPD subgroups including those with mild disease and those hospitalised with an acute exacerbation. Nonetheless, this is the first study to have examined EWGSOP defined sarcopenia in COPD. We also acknowledge debate around the use of BIA for the assessment of muscle mass, as compared with alternative methods, such as dual-energy X-ray absorptiometry (DEXA) or MRI. Previous studies have shown that BIA underestimates muscle mass compared with DEXA. ${ }^{25}$ Our cut-off values also arose from international cohorts, and their application to a UK setting requires testing. However, this offers a simple, portable assessment that was considered acceptable by the EWGSOP. Our data also raise an exemplary issue in the assessment of sarcopenia in chronic diseases. Specifically, while a low SMI is (within technical limitations) incontrovertible evidence of low muscle mass, low function may reflect both the primary disease process as well as secondary muscle dysfunction.
This may overestimate the prevalence of EWGSOP defined sarcopenia, and in that context, we note that prevalence increased with spirometrically-defined disease severity.

\section{Significance of the findings}

This is the first study to examine sarcopenia in COPD using EWGSOP criteria. Previous studies in community-dwelling and hospitalised older adults report lower overall prevalence rates ${ }^{26-29}$ than found in our cohort. Patel et $a l^{26}$ reported an overall prevalence of $6.8 \%$ and $7.8 \%$ in community-dwelling older adults using DEXA to define low muscle mass. The Belgian BELFRAIL study reported prevalence in 288 adults aged $\geq 80$ years and found that $12.5 \%$ were sarcopenic, ${ }^{27}$ similar to the prevalence in our COPD cohort, who had a mean age of 71 years. We demonstrated an association between the presence of sarcopenia and disease severity according to GOLD stage and composite prognostic indices. No previous studies have examined this relationship; though our observation is in keeping with Schols et al, ${ }^{30}$ who found a low body mass index (BMI) $\left(<21 \mathrm{~kg} / \mathrm{m}^{2}\right)$ and a low Fat-Free Mass Index ( $<16 / 15$ for males/females) was more prevalent in patients with stage IV compared with stages II-III disease.

There was no difference in prevalence of sarcopenia between patients with or without quadriceps weakness, and one-third of sarcopenic patients had preserved quadriceps strength. In both regards, sarcopenia appears to be a distinct phenotype, which cannot be identified by generalised wasting or localised weakness alone. Our findings also suggest that loss of quadriceps strength may precede loss of whole-body muscle mass in COPD. Specifically, the prevalence of quadriceps weakness was high (315 of 554 patients in whom it was measured), but of these, only 47 patients with quadriceps weakness met EWGSOP criteria for sarcopenia, while an additional 33 met the criteria without evidence of quadriceps weakness. Multiple aetiologies underlie the muscle dysfunction observed in COPD, which is characterised by atrophy, weakness and a shift towards a less oxidative fibre-type profile. ${ }^{9}$ Physical inactivity is a generally agreed precipitating factor and patients with sarcopenia had the lowest daily step count and energy expenditure based on self-reported and objective assessments. Other aetiological factors, such as hypoxaemia, inflammation, medication, may also have an adverse effect on the muscular system, and contribute to the sarcopenic state. ${ }^{10}$

Few studies have investigated a differential response to rehabilitation in patients with sarcopenia. Liu $e t a l^{31}$ followed older adults with mobility disabilities undertaking a 12 to 18-month exercise programme and found no significant difference in the adherence rate of patients with and patients without sarcopenia $(74 \%$ vs $71 \%$, respectively, $\mathrm{p}=0.59)$. COPD patients with sarcopenia responded well to PR, with improvements in functional performance, exercise capacity, lower and upper limb strength, and health status, similar to those gained by patients without sarcopenia and exceeding known minimally important differences. ${ }^{32}{ }^{33}$ Although observed improvements were equivalent, patients with sarcopenia started PR from a lower baseline; they were weaker, had a lower BMI, reduced exercise capacity and functional performance. To this end, we were able to demonstrate that PR can reverse sarcopenia in select patients, in particular, those with an SMI or functional performance that was close to the cut-off threshold at baseline. The reversal of the state in $12 / 43(28 \%)$ patients was helped by the EWGSOP diagnostic criteria; an improvement in either SMI or an aspect of function across a cut-off led to a state reversal. In this respect, the criteria are operationally more likely to show benefit from exercise and nutritional interventions, which impact favourably on function as well as muscle mass. Nonetheless, a larger proportion of patients 
maintained their sarcopenia status, supporting a role for testing pharmacological adjuncts in selected patients.

The risk associated with sarcopenia in COPD has not yet been studied, and future longitudinal studies are required to examine its impact on adverse events and survival. In older nursing-home residents, Landi et $a l^{34}$ demonstrated that sarcopenia was associated with increased risk of all-cause mortality, and in a separate cohort of acutely unwell older patients, sarcopenia increased the risk of non-elective readmission and mortality. ${ }^{13}$ Aspects of muscle dysfunction and low functional performance have previously been related to adverse outcomes in COPD,$^{5-7} 35$ though the serious consequences of the sarcopenia syndrome warrant further research to establish the risk it carries in patients with COPD. ${ }^{29} 30$

In conclusion, there is a $15 \%$ prevalence of sarcopenia in patients with COPD. The sarcopenia phenotype is associated with reduced function, exercise capacity and health status, and is distinct from localised quadriceps weakness. Sarcopenia does not appear to impact on response to PR in COPD, which can lead to a reversal of the syndrome in select patients.

Contributors WD-CM is the overall guarantor of this manuscript and takes responsibility for the content, including the data and analysis. The original study conception was by WD-CM and MM. SEJ, SSCK, JLC, CMN and ALC contributed to data acquisition. SEJ and MM undertook the initial analysis of data, which was then reviewed by WD-CM and MIP. SEJ and MM produced a first draft of the manuscript. All authors critically revised the manuscript and approved the submitted version.

Funding SEJ, JLC and MIP are fully or partly funded by the NIHR Respiratory Biomedical Research Unit at the Royal Brompton and Harefield NHS Foundation Trust and Imperial College London. MM is supported by a National Institute for Health Research (NIHR) Post-Doctoral and a Clinical Trials Fellowship. SSCK is supported by the Medical Research Council. CMN is supported by a NIHR Doctoral Fellowship. WD-CM is supported by a National Institute for Health Research Clinician Scientist Award (CS/7/007), a Medical Research Council (UK) New Investigator Research Grant (G1002113) and a National Institute for Health Research Clinical Trials Fellowship (NIHR-CTF-01-12-04). This project was undertaken at the NIHR Respiratory Biomedical Research Unit at the Royal Brompton and Harefield NHS Foundation Trust and Imperial College London. The views expressed in this publication are those of the authors and not necessarily those of the NHS, the NIHR nor the Department of Health.

Competing interests MIP has received personal reimbursement for lecturing or consultancy regarding muscle function in COPD from Novartis and Philips Respironics; he discloses institutional reimbursement for consultancy from GlaxoSmithKline, Novartis, Regeneron, Lilly, Biomarin and Boehringer Ingelheim and institutional agreements to do research with GlaxoSmithKline, Novartis, AstraZeneca and Philips Respironics. All other authors declare that they have no conflicts of interest.

Ethics approval West London (11/H0707/2) and London Camberwell St Giles (11/L0/1780) research ethics committees.

Provenance and peer review Not commissioned; externally peer reviewed.

Data sharing statement There are no unpublished data from this study. Additional data can be found in the online supplement.

\section{REFERENCES}

1 Maltais F, Decramer M, Casaburi R, et al. An official American Thoracic Society/ European Respiratory Society statement: update on limb muscle dysfunction in chronic obstructive pulmonary disease. Am J Respir Crit Care Med 2014;189:e15-62.

2 Seymour JM, Spruit MA, Hopkinson NS, et al. The prevalence of quadriceps weakness in COPD and the relationship with disease severity. Eur Respir J 2010;36:81-8.

3 Shrikrishna D, Patel M, Tanner RJ, et al. Quadriceps wasting and physical inactivity in patients with COPD. Eur Respir J 2012;40:1115-22.

4 Natanek SA, Gosker HR, Slot IG, et al. Heterogeneity of quadriceps muscle phenotype in chronic obstructive pulmonary disease (COPD); implications for stratified medicine? Muscle Nerve 2013:48:488-97.

5 Swallow EB, Reyes D, Hopkinson NS, et al. Quadriceps strength predicts mortality in patients with moderate to severe chronic obstructive pulmonary disease. Thorax 2007:62:115-20.

6 Marquis K, Debigare R, Lacasse $Y$, et al. Midthigh muscle cross-sectional area is a better predictor of mortality than body mass index in patients with chronic obstructive pulmonary disease. Am J Respir Crit Care Med 2002;166:809-13.
7 Patel MS, Natanek SA, Stratakos G, et al. Vastus lateralis fiber shift is an independent predictor of mortality in chronic obstructive pulmonary disease. Am J Respir Crit Care Med 2014;190:350-2.

8 Cruz-Jentoft AJ, Baeyens JP, Bauer JM, et al. Sarcopenia: European consensus on definition and diagnosis: Report of the European Working Group on Sarcopenia in Older People. Age Ageing 2010;39:412-23.

9 Kalyani RR, Corriere M, Ferrucci L. Age-related and disease-related muscle loss: the effect of diabetes, obesity, and other diseases. Lancet Diabetes Endocrinol 2014:2:819-29.

10 Donaldson AV, Maddocks M, Martolini D, et al. Muscle function in COPD: a complex interplay. Int J Chron Obstruct Pulmon Dis 2012;7:523-35.

11 Spruit MA, Watkins ML, Edwards LD, et al. Determinants of poor 6-min walking distance in patients with COPD: the ECLIPSE cohort. Respir Med 2010;104:849-57.

12 Volpato S, Bianchi L, Cherubini A, et al. Prevalence and clinical correlates of sarcopenia in community-dwelling older people: application of the EWGSOP definition and diagnostic algorithm. J Gerontol A Biol Sci Med Sci 2014;69:438-46.

13 Gariballa S, Alessa A. Sarcopenia: prevalence and prognostic significance in hospitalized patients. Clinical Nutrition 2013:32:772-6.

14 Vetrano DL, Landi F, Volpato S, et al. Association of Sarcopenia With Short- and Long-term Mortality in Older Adults Admitted to Acute Care Wards: Results From the CRIME Study. J Gerontol A Biol Sci Med Sci 2014;69:1154-61.

15 Steiner MC. Sarcopaenia in chronic obstructive pulmonary disease. Thorax 2007:62:101-3.

16 Schols AM, Ferreira IM, Franssen FM, et al. Nutritional assessment and therapy in COPD: a European Respiratory Society statement. Eur Respir J 2014;44:1504-20. pii: erj00709-2014.

17 Steiner MC, Roubenoff $\mathrm{R}$, Tal-Singer $\mathrm{R}$, et al. Prospects for the development of effective pharmacotherapy targeted at the skeletal muscles in chronic obstructive pulmonary disease: a translational review. Thorax 2012:67:1102-9.

18 Kon SS, Patel MS, Canavan JL, et al. Reliability and validity of 4-metre gait speed in COPD. Eur Respir J 2013;42:333-40.

19 Kon SS, Canavan JL, Nolan CN, et al. The 4-metre gait speed in COPD: responsiveness and minimal clinically important difference. Eur Respir J 2014:43:1298-305.

20 Janssen I, Baumgartner RN, Ross R, et al. Skeletal muscle cutpoints associated with elevated physical disability risk in older men and women. Am J Epidemiol 2004;159:413-21.

21 Lauretani F, Russo CR, Bandinelli S, et al. Age-associated changes in skeletal muscles and their effect on mobility: an operational diagnosis of sarcopenia. J Appl Physiol (1985) 2003;95:1851-60.

22 Garfield BE, Canavan JL, Smith CJ, et al. Stanford Seven-Day Physical Activity Recall questionnaire in COPD. Eur Respir J 2012;40:356-62.

23 Rosenbaum $P$, Rubin $D$. The central role of the propensity score in observational studies for causal effects. Biometrika 1983:70:41-55.

24 Morley JE, Abbatecola AM, Argiles JM, et al. Sarcopenia with limited mobility: an international consensus. J Am Med Dir Assoc 2011;12:403-9.

25 Steiner MC, Barton RL, Singh SJ, et al. Bedside methods versus dual energy $\mathrm{X}$-ray absorptiometry for body composition measurement in COPD. Eur Respir $J$ 2002;19:626-31.

26 Patel HP, Syddall HE, Jameson $\mathrm{K}$, et al. Prevalence of sarcopenia in communitydwelling older people in the UK using the European Working Group on Sarcopenia in Older People (EWGSOP) definition: findings from the Hertfordshire Cohort Study (HCS). Age Ageing 2013;42:378-84.

27 Legrand $D$, Vaes $B$, Mathei $C$, et al. The prevalence of sarcopenia in very old individuals according to the European consensus definition: insights from the BELFRAIL study. Age Ageing 2013;42:727-34.

28 Smoliner C, Sieber CC, Wirth R. Prevalence of sarcopenia in geriatric hospitalized patients. J Am Med Dir Assoc 2014;15:267-72.

29 Rossi $A P$, Fantin $F$, Micciolo $R$, et al. Identifying sarcopenia in acute care setting patients. J Am Med Dir Assoc 2014;15:303 e7-12.

30 Schols AM, Broekhuizen R, Weling-Scheepers CA, et al. Body composition and mortality in chronic obstructive pulmonary disease. Am J Clin Nutr 2005;82:53-9.

31 Liu CK, Leng $X$, Hsu FC, et al. The impact of sarcopenia on a physical activity intervention: the Lifestyle Interventions and Independence for Elders Pilot Study (LIFE-P). J Nutr Health Aging 2014;18:59-64.

32 Kon SS, Canavan JL, Jones SE, et al. Minimum clinically important difference for the COPD Assessment Test: a prospective analysis. Lancet Respiratory Medicine 2014;2:195-203.

33 Singh SJ, Puhan MA, Andrianopoulos V, et al. An official systematic review of the European Respiratory Society/American Thoracic Society: measurement properties of field walking tests in chronic respiratory disease. Eur Respir J 2014;44:1447-78. pii: erj01504-2014.

34 Landi F, Cruz-Jentoft AJ, Liperoti $R$, et al. Sarcopenia and mortality risk in frail older persons aged 80 years and older: results from ilSIRENTE study. Age Ageing 2013:42:203-9.

35 Polkey MI, Spruit MA, Edwards LD, et al. Six-minute-walk test in chronic obstructive pulmonary disease: minimal clinically important difference for death or hospitalization. Am J Respir Crit Care Med 2013;187:382-6. 